\title{
Self-reported discriminatory and positive behaviours towards people with mental health problems: Findings from an Australian national survey
}

Running title: Self-reported discrimination towards people with mental health problems

Nicola J Reavley, Principal Research Fellow

Amy J Morgan, Research Fellow

Alyssia Rossetto, Research Fellow

Anthony F Jorm, Professorial Fellow

Centre for Mental Health, Melbourne School of Population and Global Health, The University of Melbourne, Australia

Corresponding author:

Nicola J Reavley, Centre for Mental Health, Melbourne School of Population and Global Health, 207 Bouverie Street, VIC 3010, The University of Melbourne, Australia.

Email: nreavley@unimelb.edu.au, P: +61 390357628 F: +61 393495815

\section{Funding}

The study was funded by the National Health and Medical Research Council. 


\section{Self-reported discriminatory and positive behaviours towards people with mental health problems: Findings from an Australian national}

\section{survey}

\section{Abstract}

\section{Objective}

The aim of the study was to explore self-reported avoidance, discrimination and positive treatment by members of the public towards people with mental health problems.

\section{Methods}

In 2014, telephone interviews were carried out with 5220 Australians aged 18+. Respondents were asked if they had known an adult with a mental health problem over the previous 12 months. If they had, they were asked further questions about the person's age, gender, relationship to the respondent and their mental health problem. Respondents were then asked if they had avoided, discriminated against or treated the person more positively and, if so, some details about what happened.

\section{Results}

$19.9 \%$ of respondents reported avoiding someone with a mental health problem, with the most common reasons being difficulty tolerating the person's behaviour and needing time out. However, respondents were more likely to report treating the person with mental health problems more positively $(73.0 \%)$ than avoiding or discriminating against them (4.7\%). The most common positive behaviours were non-specific support and maintaining or increasing contact. Avoidance was less likely from friends and those aged 60+. Discrimination was more likely from family members and spouses and less likely from respondents aged 60+. Positive treatment was more likely from people who had experienced a mental health problem. 


\section{Conclusions}

This study provides insight into the reasons why people avoid others with mental health problems.

The results can provide input into the design of anti-discrimination interventions and further

empower people with mental health problems as they advocate for change in the area of discrimination.

Key words: mental disorders, discrimination, stigma, positive treatment 


\section{Background}

Discrimination may profoundly impact the life of a person with a mental health problem. It is a barrier to social participation, limiting personal relationships and the ability to achieve educational and vocational goals [1,2]. It may also worsen psychological distress, inhibit help seeking and reduce treatment adherence $[3,4]$.

While the majority of studies in the area of stigma have involved the assessment of attitudes towards people with mental health problems, a number of recent studies have explored the experiences of people with these problems, most commonly in clinical populations or people with one specific disorder (most commonly schizophrenia) [5-8]. Population-based surveys have found mean prevalence estimates of unfair treatment of between $16 \%$ and $37 \%$ in high-income countries

[9-11]. Yet, in spite of these relatively high prevalence estimates, there has been no systematic study of those who behave in a discriminatory or more supportive way towards people with mental health problems.

In 2014 an Australian population-based survey was conducted, the National Survey of Discrimination and Positive Treatment Associated with Mental Health Problems [12]. The survey involved 5220 Australians aged 18+, 1381 of whom reported a mental health problem or scored highly on a symptom screening questionnaire. Questions covered experiences of avoidance, discrimination and positive treatment by friends, spouse, other family, workplace, educational institution and others in the community. The results showed that in most domains, respondents reported more positive treatment experiences than avoidance or discrimination, e.g., 50\% of respondents reported being treated more positively by friends while $22 \%$ reported being avoided and $14 \%$ reported being discriminated against. The only domain in which discrimination occurred more frequently than positive treatment was in looking for work [13].

Given the relatively high prevalence of both discrimination and positive treatment, it is striking that there have been no studies directly asking about the experiences of those who have either 
discriminated against people with mental health problems or treated them more positively. However, in the absence of existing literature on people's own reports of their discriminatory or supportive behaviours towards people with mental health problems, it may be useful to look at existing literature on self-reported behaviours towards other groups of people who may be stigmatised, including people from the Lesbian, Gay, Bisexual, Transgender, Intersex (LGBTI) community. LGBTI individuals, similar to people with mental health problems, may choose to conceal their potentially stigmatised characteristics. A small number of studies have explored discriminatory behaviour towards gay people, finding high prevalence of discriminatory behaviour, with older people and those who had less direct contact with gays and lesbians more prone to discriminate against them [14-16].

However, there are limitations in comparing research into discrimination against LGBTI people with that against people with mental health problems, as there is evidence that prejudices and stereotypes are different $[14,16]$. There is therefore a need for research to better understand the ways in which people characterise their behaviour towards people with mental health problems and to understand the reasons and determinants of those behaviours. This study, which is part of a broader study into experiences of discrimination and positive treatment of people with mental health problems, aims to shed light on these questions.

\section{Methods}

This paper reports data from the National Survey of Discrimination Associated with Mental Health Problems, which surveyed Australians about experiences of discrimination and positive treatment related to mental health problems. For detailed survey methodology see Reavley and Jorm [12]. Briefly, the survey involved computer-assisted telephone interviews (CATI) with a national sample of 5220 members of the general community aged 18 years and over. The sample was contacted by random-digit dialling of both landlines and mobile phones (dual frame design). Interviews were conducted between October and December 2014 by the Social Research Centre survey company. 
The average interview length was 19.4 minutes. Ethics approval was obtained from the University of Melbourne Human Research Ethics Committee.

\section{Survey interview}

Initial questions covered sociodemographic information (age, gender, marital status, postcode, country of birth, language spoken at home, level of education and Aboriginal and Torres Strait Islander status). It also included questions about the respondent's own experience of mental health problems defined in the following way: "By a 'mental health problem' we mean a period of weeks or more when you are feeling depressed, anxious, or emotionally stressed, and these problems are interfering with your life. Mental health problems could include, for example, depression, anxiety disorders, eating disorders, schizophrenia, bipolar disorder, or personality disorders". They were then asked whether they had experienced avoidance, discrimination and positive treatment (see $[17,18,12,13]$ for further details).

Respondents were then asked whether they knew someone else with mental health problems. Those who reported definitely knowing someone with a mental health problem were asked if they knew more than one person. In such cases, they were asked to think about the person they knew best. They were asked about the mental health problem the person had, how they knew the person had the problem, and the person's age, gender and relationship to the respondent. Those who knew someone with any of the following mental health problems were considered in scope: depression/major depression, attempted suicide or self-harm , anxiety/anxiety disorder, posttraumatic stress disorder/PTSD, agoraphobia, panic disorder, obsessive-compulsive disorder/OCD, social phobia, generalised anxiety disorder/GAD, eating disorder/ anorexia/bulimia, schizophrenia/paranoid schizophrenia, schizoaffective disorder, psychosis/psychotic, bipolar/bipolar disorder/manic-depressive disorder, mental illness, personality disorder/borderline personality disorder, attention deficit-hyperactivity disorder/ADHD, Autism/Asperger's and nervous breakdown. Respondents were then asked if, in the last 12 months, they themselves had avoided 
the person or anyone else with a mental health problem, treated the person or anyone else unfairly, or treated the person or anyone else more positively because of their mental health problems.

Those who answered in the affirmative were asked to describe what happened.

\section{Statistical analysis}

The data were analysed using percent frequencies and $95 \%$ confidence intervals. A pre-weight was applied to adjust for the dual frame design and the respondent chance of selection. The achieved sample was close to the Australian national population in terms of geographic distribution; however, there was an under-representation of males and of younger adults, and an over-representation of university-educated individuals and people with an English-speaking background. These biases were adjusted for by 'raking' (also known as rim weighting or iterative proportional fitting) to account for known population proportions of gender, age, education level, region and telephone status, with age and gender based on Australian Bureau of Statistics (ABS) estimated population data from March 2014 [19], level of education and region based on ABS 2011 census data [20] and telephone status based on 2011 Australian Communication and Media Authority data [21]).

Associations between respondent characteristics (sociodemographic and own experience of mental health problem), characteristics of the person known to the respondent (gender and relationship to respondent) and respondent avoidance, discrimination or positive treatment were assessed with multiple logistic regression. The dependent variables were coded into two categories: yes vs no. Covariates were gender; age (coded into 3 groups: $18-29$ years, 30-59 years, $60+$ years); language spoken at home (other vs English); level of education (bachelor or above vs below bachelor); having experienced a mental health problem (yes vs no); gender of person known to respondent; category of mental disorder of the person known to the respondent: depression, anxiety disorder (including PTSD and OCD), bipolar disorder, any other disorder (including attempted suicide or self-harm, eating disorder, schizophrenia, schizoaffective disorder, psychosis/psychotic, personality disorder, ADHD, Autism/Asperger's, nervous breakdown, mental illness) and relationship to respondent 
(multiples accepted). For the last variable, only those categories into which more than $5 \%$ of the sample fell were included, namely family member, friend, spouse or work colleague. All analyses were performed using Intercooled Stata 13 (StataCorp LP, Texas, USA).

\section{Content analysis}

The open-ended verbatim responses for each question were contained in three datasets. Each coding system followed the same development process. The responses in each data set were coded and categorised using the content analysis principles outlined in Crowe et al [22]. One author (A.R.) read all the responses to a given question and drafted a coding system. This system contained instructions, examples and counterexamples from the data set. It was partly based on existing coding frameworks that were developed as part of this research (described elsewhere, e.g., $[17,13])$. A random sample of responses (between 6 and 10\% depending on whether the responses were given to the question about avoidance, discrimination or positive treatment) was then independently double-coded by a second author (A.J.M.) to test and refine the coding system. Agreement was then evaluated and any coding discrepancies were resolved by discussion between the coders and, where necessary, a third author (N.J.R.). Changes to the coding system's instructions and/or categories were also made during this process. Once the coding system was finalised, one author (A.R.) coded the remaining responses in the data set, discussing any that were difficult to code with a second author. Responses could be coded into more than one category. Responses which could not be interpreted, did not make sense within the context of the question or required the coder to make strong assumptions about the participant's meaning were categorised as "unable to code" and excluded from subsequent analyses. Only categories with frequencies greater than $5 \%$ are reported below.

\section{Results}

Overall, 5220 interviews were completed, with 2589 on landlines and 2631 on mobiles. The standard response rate for the survey was $37.5 \%$ and the American Association of Public Opinion Research 
(AAPOR) response rate (which enables a more accurate assessment of the response rate, as some households that refuse the screening process would in fact be in scope) was $18.5 \%$. The sample characteristics are given in Table 1.

Table 2 shows respondents' treatment of other people with mental health problems. Respondents reported that their positive treatment of other people with mental health problems was more frequent than avoidance, which was more frequent than discriminatory treatment.

Qualitative content analysis led to the description of broad categories of experiences for each of the following domains (totals may add to more than $100 \%$, as responses could fit in multiple categories): avoidance, discrimination and positive treatment. Examples from each category are provided for illustration, with the respondent's gender, age group and the mental health problem(s) of the person they knew given after each quote.

\section{Reasons for avoidance}

Difficulty managing/tolerating person's symptoms/behaviour: 187 respondents (36\%) mentioned that the person's symptoms, behaviours or mental health were difficult to manage, or caused concern, annoyance or frustration for the respondent.

Because she could get quite cranky and sort of angry. Sometimes she would accuse you of something you hadn't done. She also upset one of my friends. (Female, age range 60-65, reporting on a person with bipolar disorder)

Didn't like the person or their behaviour. (Male, 70-74, reporting on a person with depression)

I avoided them because they irritated me but I don't know if it was just part of the person. (Female, 25-29, reporting on a person with depression/bipolar disorder) 
I couldn't stand the negativity that they expressed when I was around them. (Female, 35-39, reporting on a person with depression)

Needing time out: 151 respondents (29\%) mentioned needing a break, space or time out from supporting the person, including having to take care of their own mental health or other problems.

I exhausted myself, carer's fatigue, energy levels, in some ways self-preservation. I was supporting a partner who was involved in the situation, it was tiring. (Female, 25-29, reporting on a person with anxiety disorder)

For my own mental health, mostly for my own sanity. I do as much as I can but sometimes I need a break. (Female, 55-59, reporting on a person with schizophrenia)

I am highly stressed person already and don't think I could handle someone else problems on top of mine, mainly the stress, not sure what to tell them. (Female, 25-29, reporting on a person with bipolar disorder)

Person was dangerous/aggressive: 90 respondents (17\%) reported that the person was a danger to themselves, others or the respondent, was aggressive, abusive or violent, or the respondent felt unsafe around them.

Collecting for a charity, just didn't knock on his door. Knew of his aggression (Female, 55-59, reporting on a person with an unspecified problem)

Because they bullied me, I tried to have as little to do with them as possible (Female, 45-49, reporting on a person with depression/personality disorder)

Because she has threatened me and I felt very unsafe in her presence (Female, 50-54, reporting on a person with personality disorder)

Avoidance was best/easiest: 62 respondents (12\%) reported that it was best to avoid the person or it was too hard to deal with them. 
Too problematic to deal with them. (Male, 75+, reporting on a person with bipolar disorder) Just in the work environment, it was just easier when they were really unwell. (Female, 3034 , reporting on a person with bipolar disorder)

Difficulty in communicating with the person. (Male, 25-29, reporting on a person with depression)

Person didn't want help: 37 respondents (7\%) said that the person wanted to deal with their problems alone or did not respond positively to the support offered.

He told me he no longer wanted to associate with me. (Male, 45-49, reporting on a person with depression)

When they're in a depressed state they just want to be left alone so you just leave them to it. They just want to be alone. They don't want you. They just want to be alone and then they'll come right. (Female, 45-49, reporting on a person with depression)

There is just no way that I can help. It becomes very difficult to communicate. Constantly the same conversations, I don't feel she is helping. Depression going on for long time, not recent. She self-medicates herself. She doesn't follow doctors' orders. Goes to internet. She doesn't understand. I feel there is no way to be able to help her. (Female, 75+, reporting on a person with depression)

Because of his mental health problem and living at home, I set down ground rules for living at home but he wouldn't abide by these rules so I had to ask him to live somewhere else. (Female, 55-59, reporting on a person with depression/anxiety disorder)

Relationship breakdown or conflict: 38 respondents (7\%) reported avoiding the person due to relationship breakdown or conflict. 
She was my partner at the time and I didn't want to argue with her. (Male, 30-34, reporting on a person with bipolar disorder)

It takes its toll to a degree. It's avoidance but her mental health has had a negative effect on our relationship. (Male, 45-49, reporting on a person with depression/anxiety disorder/bipolar disorder)

Respondent couldn't help: 26 respondents (5\%) felt unable to help the person or that their presence would make things worse.

Sometimes when she is in a dark place I can't really help her so I sort of avoid her because I know I cannot help her. (Female, 50-54, reporting on a person with depression/anxiety disorder)

Not knowing how to manage it, not knowing how to help, getting frustrated by the person. (Female, 25-29, reporting on a person with depression/anxiety disorder)

\section{Reports of discrimination}

Reducing/cutting off contact: 37 respondents (36\%) reported reducing contact with the person.

I ceased to be around that person. (Male, 45-49, reporting on a person with bipolar disorder)

Could've been more understanding to his mental situation. I did give him a couple of chances, but unfortunately the last straw broke the camel's back. We warned him to do some things but he didn't. Ended up having to remove him from our household. (Male, 3539, reporting on a person with bipolar disorder)

Anger/arguments/frustration: 25 respondents (25\%) reported getting "angry", "frustrated", or "impatient" with the person (or similar terms).

Because they can really get a bit aggressive. I guess they can't control it. Instead of defusing the situation, I argue back. I don't think properly, I kind of forget and I get a bit angry. Then I 
realise that it is not right. Try and cool things down. (Female, 40-44, reporting on a person with anxiety disorder)

Lack of support/respect: 12 respondents (12\%) reported not providing the person with appropriate or necessary support, despite knowing that the person was ill.

I haven't been as supportive as I should have been and stepped in when I should have.

(Male, 55-59, reporting on a person with anxiety disorder/PTSD)

I guess I'm not as nice or respectful as I would be to others when she's just had enough of them. I'm the primary carer for my dad, her and brother. I get frustrated and could be a bit unfair. (Female, 35-39, reporting on a person with schizophrenia)

Lack of awareness or understanding: 11 respondents (11\%) mentioned not understanding the person's illness or being aware of their mental health problems, or the extent of it.

At some stages, just lack of awareness of what her condition actually entailed. (Female, 3034, reporting on a person with bipolar disorder/personality disorder/drug addiction)

Being dismissive: 6 respondents (6\%) mentioned dismissing that the person's illness was real, or that it was serious and caused suffering.

To be honest I don't really believe in mental health problems, like I do to a certain extent but when it comes to anxiety I believe it's a bit of a load of <expletive removed> and get over it and get on with life just like everyone else does. (Male, 18-19, reporting on a person with depression/anxiety disorder)

\section{Reports of positive treatment}

Non-specific support: 986 respondents (50\%) mentioned being supportive or more supportive than usual. 
Being a little more helpful, being more supportive. (Female, 45-49, reporting on a person with depression)

... being polite and kind to her so we give her the same respect... (Male, 55-59, reporting on a person with bipolar disorder)

Because of the situation I'm in at work, I treat most people more positively without sickening or overly enthusiastic, there is a fine line. (Male, 50-54, reporting on a person with schizophrenia/bipolar disorder)

Maintain/increase contact: 775 respondents (40\%) maintained or increased contact with the person.

Being in contact every day and putting other stuff on one side to help and offering help. (Male, 40-44, reporting on a person with depression)

By helping him out. Working out times that he can see the kids and giving him somewhere to stay when he comes up here. (Female, 40-44, reporting on a person with depression/anxiety disorder)

I got the family to rally around, and we were all aware that he was struggling with depression. (Male, 60-64, reporting on a person with depression)

Became closer to them because I know what they're going through. (Male, 18-19, reporting on a person with depression)

Adjusts expectations/makes allowances: 262 respondents (13\%) reported recognising that the person had a problem, and adjusting their expectations of what the person could do.

I guess I'd be more accepting of their behaviour than if they didn't have a mental health problem. (Female, 25-29, reporting on a person with depression/anxiety disorder) 
Acceptance of normally unacceptable behaviour. (Male, 40-44, reporting on a person with schizophrenia)

Adjusting work expectations, that sort of thing, limited some responsibilities in the workplace, managing it so that they are doing manageable levels of work and not overwhelmed. (Male, 55-59, reporting on a person with depression/social phobia)

Giving them some leeway in the job or they need time off or didn't start as early. (Male, 6569 , reporting on a person with depression)

I can recognise he can't initiate things for his business so I set the website up. (Female, 4549 , reporting on a person with psychosis)

Try to cheer up: 233 respondents (12\%) reported trying to cheer the person up.

By being there for them and making them feel happy and making them smile. (Female, 2529 , reporting on a person with depression)

Always trying to be a little more upbeat around her. If everyone is more happy around her she tends to be more happy. Stuff like that. (Male, 20-24, reporting on a person with depression)

Practical assistance with daily tasks: 166 respondents (8\%) mentioned assisting the person with daily tasks, e.g. housework/chores, food preparation, providing transport or walking pets.

... did her shopping, and took her shopping when she could go in her wheel chairs. (Female, 70-74, reporting on a person with depression)

...we also give help with caring of the children and babysitting so that they can attend the meeting that support them. (Female, 60-64, reporting on a person with anxiety disorder/eating disorder) 
...I've also had to take her to the doctors, I try to be there for her... (Female, 55-59, reporting on a person with schizophrenia)

Checking on them: 119 respondents (6\%) mentioned checking on the person to see how they were going and whether they were okay.

Always making sure they're okay and checking up on them. (Female, 18-19, reporting on a person with bipolar disorder)

Because I knew what it was like, I always made sure she was fine, and dropped around to her house and talk for hours. (Male, 18-19, reporting on a person with depression)

Talk/listen: 108 respondents (6\%) reported being able to talk with the person about their issues/feelings/mental health problems, or the respondent listened/was willing to listen to the person talk about their issues.

Basically just chat to him about the issues and see if I can be of any help - over the phone and face to face. (Male, 45-49, reporting on a person with depression)

Discuss my personal information including my medication, advised him how it helped me. (Male, 50-54, reporting on a person with depression)

Encourage professional help: 97 respondents (5\%) reported encouraging or assisting the person to seek help or professional help for their problem.

Assisted them to get a mental health plan. (Male, 40-44, reporting on a person with depression)

After the episode where she ended up in the mental health unit I went there every day to see her, I brought her food from outside, I talked to the psychologist there for her. (Female, 60-64, reporting on a person with anxiety disorder) 


\section{Predictors of self-reported avoidance, discrimination and positive treatment}

Overall there were few consistent predictors of respondent avoidance, discrimination and positive treatment. Respondents aged 60+ were less likely to avoid and discriminate against someone they knew with a mental health problem (see Table 3). Friends were less likely to avoid the person and family members and spouses were more likely to discriminate against the person. Respondents were more likely to treat a person with depression more positively than people with other kinds of mental health problems.

\section{Discussion}

This paper reports results of the first national population-based survey to assess how respondents treat people they know who have mental health problems. This is of value because, while a person with mental health problems may describe their experiences, only the person acting in a discriminatory or supportive way can give their reasons for these behaviours. The results of this study showed a greater frequency of reported positive treatment than avoidance or discrimination. While this pattern mirrors that of experiences described by respondents of the survey who had mental health problems, in this case, the frequencies of discrimination were much lower and the frequencies of support were much higher, e.g. $50 \%$ of people reported being treated more positively by friends while $73 \%$ of respondents engaged in self-reported positive behaviours; $14 \%$ of people reported discrimination from friends while $5 \%$ of respondents engaged in self-reported discrimination [12]. Social desirability may also have influenced responses to the questions about self-reported behaviour, with people less willing to admit avoidance or discrimination.

These findings may reflect the difficulty in interpreting another person's actions as 'discrimination' or 'positive treatment'. There is evidence of relatively high levels of anticipated discrimination in people with mental health problems, and it is possible that a person with a mental health problem may interpret another person's actions as discrimination when someone who does not have a mental health problem would not $[6,7]$. However, previous results from this survey showed that 
when respondents reported on how a person that they knew with a mental health problem was treated by others, the frequencies were close to those that people with mental health problems themselves reported [12]. It is also possible that people act in ways that are discriminatory without necessarily realising or intending to, perhaps due to underlying assumptions about the capacities of people with mental health problems. Anti-stigma campaigns that directly focus on such behaviours may play a role in overcoming discrimination $[23,24]$.

Having difficulty tolerating the person's symptoms and behaviour or needing time out were the most commonly mentioned reasons for avoiding the person. This is also reflected in the discriminatory behaviours, for which the most commonly mentioned category was reducing or cutting off contact. In the context of the often considerable responsibility that close family members or friends of a person with severe mental health problems take on, this is not surprising $[25,26]$. The findings indicate that avoidance was largely due to the behaviour of the person the respondent knew, rather than to stereotypes or misinformation about people with mental health problems. This suggests a need for education that incorporates both acknowledgement that supporting a person with a mental health problem may be difficult and advice on how to provide appropriate support.

These findings mirror the experiences described by respondents of the survey who had experienced mental health problems, and by other studies of service users with mental health problems, who describe being shunned or avoided as the most common experiences $[6,5,8]$. Among the supportive actions, non-specific support and maintaining or increasing contact were the most commonly nominated behaviours. These findings are in line with a national survey of how people helped someone they knew well who developed a mental health problem [27]. The most commonly reported actions were listening to the person, providing support and information and encouraging them to seek appropriate professional help.

Analysis of predictors of avoidance, discrimination and positive treatment showed that family members and spouses were more likely to discriminate against the person, possibly because 
avoidance is more difficult in the context of these relationships. These findings point to a need for more support for family members of people with mental health problems. While resources for 'carers' are available, family members of people with common mental disorders (rather than low prevalence disorders) may not consider this role to be applicable to themselves and there may be a need for psychoeducation specifically targeting family members of people with anxiety and depressive disorders. Public education campaigns could also focus on families of people with these disorders, acknowledging the likely impact on them and giving information about how to support the person while also accessing support and practicing good self-care. Respondents were more likely to treat a person with depression more positively, which may be due to improved community mental health literacy in relation to depression [28]. Analysis also showed that older people were less likely to avoid the person and to discriminate. This is somewhat contradictory to previous research which shows that older people are more likely to desire social distance from a person with mental health problems [29]. It is possible that older people do not see their behaviours as discriminatory or have already ceased contact with those they wish to avoid.

This study has several strengths. It is the first study to assess, at a population level, the self-reported discriminatory and positive behaviours towards people with mental health problems. Limitations of the study include social desirability bias and future studies could incorporate a measure of social desirability bias to explore its association with self-reported behaviour towards those with mental health problems [30]. In addition, the relatively low response rate of $37 \%$ which, while in line with other similar Australian surveys, may limit the generalisability of the results [31]. It is also possible that while the questions specifically ask about respondent treatment of the person 'because of their mental health problems', some of the responses reflect interpersonal problems that respondents ascribe to the person's mental health or their diagnosis, but are in fact unrelated. Moreover, the most common disorders reported in those respondents knew were depression and anxiety disorders and the reasons for avoidance and discrimination may not translate to those with low prevalence disorders. 
It is hoped that the results of the current study can assist people with mental health problems and others in their social networks to better understand each other's actions and provide input into the design of anti-discrimination interventions, for example, public education about how to support someone with a mental health problem.

\section{Funding}

The study was funded by the National Health and Medical Research Council.

\section{Conflict of interest}

None identified. 


\section{References}

1. Link BG, Struening EL, Rahav M, Phelan JC, Nuttbrock L (1997) On stigma and its consequences: evidence from a longitudinal study of men with dual diagnoses of mental illness and substance abuse. J Health Soc Behav 38 (2):177-190

2. Brouwers EP, Mathijssen J, Van Bortel T, Knifton L, Wahlbeck K, Van Audenhove C, Kadri N, Chang

C, Goud BR, Ballester D, Tofoli LF, Bello R, Jorge-Monteiro MF, Zaske H, Milacic I, Ucok A, Bonetto C, Lasalvia A, Thornicroft G, Van Weeghel J, Group* AIS (2016) Discrimination in the workplace, reported by people with major depressive disorder: a cross-sectional study in 35 countries. BMJ Open 6 (2):e009961. doi:10.1136/bmjopen-2015-009961

3. Corrigan P (2004) How stigma interferes with mental health care. The American Psychologist 59 (7):614-625

4. Wells JE, Robins LN, Bushnell JA, Jarosz D, Oakley-Browne MA (1994) Perceived barriers to care in St. Louis (USA) and Christchurch (NZ): reasons for not seeking professional help for psychological distress. Soc Psychiatry Psychiatr Epidemiol 29 (4):155-164

5. Angermeyer MC, Beck M, Dietrich S, Holzinger A (2004) The stigma of mental illness: patients' anticipations and experiences. The International journal of social psychiatry 50 (2):153-162

6. Lasalvia A, Zoppei S, Van Bortel T, Bonetto C, Cristofalo D, Wahlbeck K, Bacle SV, Van Audenhove C, van Weeghel J, Reneses B, Germanavicius A, Economou M, Lanfredi M, Ando S, Sartorius N, LopezIbor JJ, Thornicroft G (2013) Global pattern of experienced and anticipated discrimination reported by people with major depressive disorder: a cross-sectional survey. Lancet 381 (9860):55-62. doi:10.1016/S0140-6736(12)61379-8

7. Thornicroft G, Brohan E, Rose D, Sartorius N, Leese M (2009) Global pattern of experienced and anticipated discrimination against people with schizophrenia: a cross-sectional survey. Lancet 373 (9661):408-415. doi:10.1016/S0140-6736(08)61817-6

8. Wahl OF (1999) Mental health consumers' experience of stigma. Schizophr Bull 25 (3):467-478 
9. Alonso J, Buron A, Bruffaerts R, He Y, Posada-Villa J, Lepine JP, Angermeyer MC, Levinson D, de Girolamo G, Tachimori H, Mneimneh ZN, Medina-Mora ME, Ormel J, Scott KM, Gureje O, Haro JM, Gluzman S, Lee S, Vilagut G, Kessler RC, Von Korff M, World Mental Health C (2008) Association of perceived stigma and mood and anxiety disorders: results from the World Mental Health Surveys. Acta psychiatrica Scandinavica 118 (4):305-314. doi:10.1111/j.1600-0447.2008.01241.x

10. Baldwin ML, Marcus SC (2006) Perceived and measured stigma among workers with serious mental illness. Psychiatric services 57 (3):388-392. doi:10.1176/appi.ps.57.3.388

11. Stuart H, Patten SB, Koller M, Modgill G, Liinamaa T (2014) Stigma in Canada: results from a rapid response survey. Canadian journal of psychiatry Revue canadienne de psychiatrie 59 (10 Suppl 1):S27-33

12. Reavley NJ, Jorm AF (2015) Experiences of discrimination and positive treatment in people with mental health problems: Findings from an Australian national survey. Australian and New Zealand Journal of Psychiatry 49 (10):906-913

13. Reavley NJ, Morgan AJ, Jorm AF (2016) Discrimination and positive treatment towards people with mental health problems in workplace and education settings: Findings from an Australian national survey. Stigma and Health (in press)

14. Franklin K (2000) Antigay behaviours among young adults: Prevalence, patterns and motivators in a noncriminal population. Journal of Interpersonal Violence 15 (4):339-362

15. Huic A, Jelic M, Kamenov Z (2015) What predicts the willingness of heterosexual persons to behave in a positive or negative manner towards homosexual persons? Criminology and Social Integration Journal $23(2): 110-141$

16. Rey AM, Gibson PR (1997) Beyond High School: Heterosexuals' Self-Reported Anti-Gay/Lesbian Behaviors and Attitudes. Journal of Gay and Lesbian Social Services 7 (4):65-84

17. Morgan AJ, Reavley NJ, Jorm AF, Beatson R (2016) Experiences of discrimination and positive treatment from health professionals: A national survey of adults with mental health problems. Aust N Z J Psychiatry 50 (8):754-762. doi:10.1177/0004867416655605 
18. Morgan AJ, Reavley NJ, Jorm AF, Beatson R (2017) Discrimination and support from friends and family members experienced by people with mental health problems: Findings from an Australian national survey. Social Psychiatry and Psychiatric Epidemiology (in press)

19. Australian Bureau of Statistics (2014) Estimated Resident Population March 2014. Australian Bureau of Statistics,, Canberra

20. Australian Bureau of Statistics (2014) ABS Census 2011 Table Builder. Australian Bureau of Statistics.

http://www.abs.gov.au/websitedbs/censushome.nsf/home/tablebuilder?opendocument\&navpos=2 $\underline{40}$.

21. Australian Communication and Media Authority (2014) Communications report 2013-14. Australian Communication and Media Authority, Canberra

22. Crowe M, Inder M, Porter R (2015) Conducting qualitative research in mental health: Thematic and content analyses. Aust N Z J Psychiatry 49 (7):616-623. doi:10.1177/0004867415582053 23. Henderson C, Thornicroft G (2013) Evaluation of the Time to Change programme in England 2008-2011. The British journal of psychiatry Supplement 55:s45-48. doi:10.1192/bjp.bp.112.112896 24. Thornicroft C, Wyllie A, Thornicroft G, Mehta N (2014) Impact of the "Like Minds, Like Mine" anti-stigma and discrimination campaign in New Zealand on anticipated and experienced discrimination. Aust N Z J Psychiatry 48 (4):360-370. doi:10.1177/0004867413512687 25. Perlick DA, Berk L, Kaczynski R, Gonzalez J, Link B, Dixon L, Grier S, Miklowitz DJ (2016) Caregiver burden as a predictor of depression among family and friends who provide care for persons with bipolar disorder. Bipolar Disord 18 (2):183-191. doi:10.1111/bdi.12379

26. Steele A, Maruyama N, Galynker I (2010) Psychiatric symptoms in caregivers of patients with bipolar disorder: a review. J Affect Disord 121 (1-2):10-21. doi:10.1016/j.jad.2009.04.020

27. Rossetto A, Jorm AF, Reavley NJ (2014) Quality of helping behaviours of members of the public towards a person with a mental illness: A descriptive analysis of data from an Australian national survey. Annals of General Psychiatry 13 (1):2 
28. Reavley NJ, Jorm AF (2012) Public recognition of mental disorders and beliefs about treatment: changes in Australia over 16 years. British Journal of Psychiatry 200:419-425

29. Jorm AF, Oh E (2009) Desire for social distance from people with mental disorders. Aust N Z J Psychiatry 43 (3):183-200. doi:908742834 [pii] 10.1080/00048670802653349

30. Henderson C, Evans-Lacko S, Flach C, Thornicroft G (2012) Responses to mental health stigma questions: the importance of social desirability and data collection method. Canadian journal of psychiatry Revue canadienne de psychiatrie 57 (3):152-160. doi:10.1177/070674371205700304

31. Curtin R, Presser S, Singer E (2005) Changes in telephone survey non-response over the past quarter century. Public Opinion Quarterly 69:87-98 


\section{Tables}

Table 1 Sample characteristics

Weighted \%

\section{Gender}

Male

Female

56.4

Age category

$18-29$ years

29.2

30-59 years

58.0

$60+$ years

12.8

\section{Highest level of education}

Below bachelor level

79.8

Bachelor degree or above

20.2

\section{Employment status}

Paid or voluntary work outside the home in past 12 months

Marital status

Married or defacto

49.2

Never married

34.8

Separated, divorced or widowed

16.0 
Language spoken at home

$\begin{array}{ll}\text { English } & 86.2\end{array}$

Other

Knew someone with an in-scope mental health problem

51.0

Mental health problem of person known to respondent

Depression

Anxiety (including PTSD, OCD)

23.8

Bipolar disorder

15.5

Personality disorder

Eating disorder

Attempted suicide or self-harm

Psychotic disorder

0.8

Autism/Asperger's

0.6

Attention deficit-hyperactivity disorder

0.5

Mental illness

Respondent's relationship with the person with a mental health problem

Family member

Friend

Spouse

Work colleague

5.7

How respondent knew the person had a mental health problem 
Person told them

Respondent recognised it

Someone else told them
61.6

50.1

23.8

Table 2 Respondent treatment of other people with mental health problems

Weighted \%

Yes, avoided 19.9

Yes, discriminated

4.7

Yes, treated more positively

73.0 
Table 3 Multiple logistic regression analyses of predictors of respondent avoidance, discrimination or positive treatment

$\begin{array}{lll}\text { Avoid } & \text { Discriminate(OR (95\% } & \text { Treat more } \\ (\mathrm{OR}(95 \% \mathrm{CI})) & \mathrm{Cl})) & \text { positively } \\ & & (\mathrm{OR}(95 \% \mathrm{Cl}))\end{array}$

Respondent gender

Female vs male

$1.06(0.83-1.36)$

$0.89(0.55-1.43)$

$1.23(0.99-1.52)$

Respondent age

$30-59$ vs $18-29$

$0.80(0.59-1.09)$

$0.89(0.49-1.59)$

$0.92(0.69-1.23)$

$60+$ vs $18-29$

$0.38(0.26-0.56)^{* * *}$

$0.28(0.13-0.61)^{* *}$

$1.12(0.81-1.55)$

Respondent language

spoken at home

Other vs English

$0.79(0.54-1.14)$

$1.37(0.72-2.60)$

$1.26(0.89-1.79)$

Respondent level of

education

Bachelor or above vs Below

$1.15(0.92-1.44)$

$0.71(0.45-1.14)$

$1.01(0.83-1.24)$

bachelor

Respondent's own mental

health problems

Yes vs No

$1.08(0.60-1.96)$

$1.25(0.41-3.79)$

$0.78(0.42-1.46)$ 
Gender of person known to

respondent

Female vs male

$0.82(0.65-1.03)$

$1.15(0.72-1.84)$

$1.07(0.87-1.33)$

Relationship of person to

respondent

Family member

$1.06(0.69-1.63)$

$3.05(1.40-6.66)^{* *}$

$1.03(0.67-1.56)$

Friend

$0.59(0.38-0.93) *$

$1.31(0.59-2.92)$

$0.93(0.6-1.43)$

Spouse

$1.24(0.73-2.10)$

$5.24(2.16-12.73)^{* * *}$

$1.53(0.89-2.65)$

Work colleague

$0.90(0.50-1.63)$

$0.75(0.21-2.69)$

$1.63(0.93-2.88)$

Mental disorder

Anxiety disorder

$0.87(0.54-1.41)$

$0.76(0.27-2.14)$

$1.38(0.83-2.31)$

Depression

$1.11(0.68-1.82)$

$0.88(0.35-2.17)$

$1.67(1.00-2.80)^{*}$

Bipolar disorder

$1.74(0.73-4.18)$

$2.25(0.58-8.74)$

1.39 (0.51-3.78)

Any other disorder

$1.46(0.72-2.94)$

$0.39(0.08-1.86)$

$1.06(0.51-2.22)$

$* * * \mathrm{p}<0.001, * * \mathrm{p}<0.01, * \mathrm{p}<0.05$ 